\title{
CARACTERIZACIÓN DEL PERFECCIONISMO DISFUNCIONAL EN LA NIÑEZ TARDÍA
}

\author{
Sonia Chemisquy ${ }^{1}$, Laura B. Oros ${ }^{123 a}$, Mónica Serppe ${ }^{23}$ y Cindy Ernst ${ }^{3}$ \\ Universidad de la Cuenca del Plata - CONICET, Posadas, Misiones, Argentina ${ }^{1}$ \\ Instituto Superior Adventista de Misiones, Leandro. N. Alem, Misiones, Argentina ${ }^{2}$ \\ Universidad Adventista del Plata. Libertador San Martín, Entre Ríos, Argentina ${ }^{3}$ \\ Orcid ID: https://orcid.org/0000-0002- 3820-3036 \\ Orcid ID: https://orcid.org/0000-0003-1265-76512 \\ Orcid ID: https://orcid.org/0000-0003-4369-8835
}

Recibido: 04 de setiembre 2018

Aceptado: 10 de diciembre 2018

\section{Resumen}

El perfeccionismo disfuncional implica la tendencia a imponerse metas y estándares muy elevados, a preocuparse excesivamente por cometer errores y a evaluar el desempeño de manera muy crítica. Este atributo puede impactar negativamente en el bienestar infantil. El objetivo de este trabajo fue describir las manifestaciones del perfeccionismo infantil disfuncional, analizando diferencias en función del género, el grado académico, el tipo de gestión escolar (pública-privada), y el lugar de procedencia. Se realizó una investigación empírica, descriptiva, de corte transversal. Participaron 1067 niños de entre 9 y 12 años de edad, alumnos de escuelas primarias, públicas y privadas, de dos provincias argentinas (Misiones y Entre Ríos). Los resultados del estudio verificaron la presencia del perfeccionismo infantil en esta franja etaria. De las manifestaciones perfeccionistas evaluadas, la que más sobresale es la autorientada, particularmente referida al ámbito escolar. Le siguen en orden de importancia el perfeccionismo socialmente prescrito y el perfeccionismo orientado a otros. El perfil más vulnerable corresponde a varones de 4to año, de escuelas públicas de la provincia de Misiones. Estos resultados revisten importancia al momento de diseñar estrategias orientadas a abordar esta problemática y a prevenir sus consecuencias negativas durante la niñez tardía.

Palabras clave: Perfeccionismo desadaptativo, niñez tardía, variables sociodemográficas.

${ }^{\mathrm{a}}$ Correspondencia al autor

E-mail: lauraorosb@gmail.com 


\title{
CHARACTERIZATION OF MALADAPTATIVE PERFECTIONISM IN LATE CHILDHOOD
}

\begin{abstract}
Maladaptive perfectionism is the tendency to self-impose unrealistic goals and stringent performance standards, to worry excessively about making mistakes, and to engage in autocritical self-evaluation. This attribute could negatively impact the psychological well-being of children. The objective of this work was to describe maladaptive perfectionism manifestations in children, and to assess its differences according to sex, school grade, kind of educational institution (public or private), and region of origin. An empiric, descriptive and cross-sectional investigation was made. Participants were 1067 children between 9 and 12 years old, students of public and private schools from two Argentinian provinces (Misiones and Entre Ríos). Results verified the presence of maladaptive perfectionism during early childhood. The most salient manifestation of perfectionism was self-oriented, especially related to school. Socially prescribed and finally other-oriented perfectionism were found next in order of importance. The most vulnerable population profile found was fourth grade children from Misiones' public schools. These results could be very important to design strategies aimed to treat this mental health problem or to prevent its negative consequences during late childhood.
\end{abstract}

Keywords: Maladaptive perfectionism, late childhood, sociodemographic variables.

\section{Introducción}

Existen ciertos problemas para la salud mental infantil que pueden llegar a pasar desapercibidos en los estudios epidemiológicos porque permanecen por debajo del umbral clínico; el perfeccionismo disfuncional, comprendido como la orientación a imponerse objetivos e ideales muy elevados, a preocuparse excesivamente por cometer errores y a evaluar el desempeño de manera muy crítica, es uno de ellos. En la escuela, el perfeccionismo puede resultar inadvertido, en especial porque algunos niños perfeccionistas intentan ocultar el malestar que experimentan porque lo perciben como una forma de fracaso (Flett \& Hewitt, 2013).

Según el modelo de Hewitt y Flett (1991), el perfeccionismo es entendido como un fenómeno multidimensional que incluye manifestaciones intra e interpersonales. El perfeccionismo orientado a uno mismo recoge las manifestaciones intrapersonales de este constructo haciendo referencia a aquellas imposiciones exageradas que se hace la persona, a la forma de reaccionar frente a situaciones de fracaso, con autoinculpación y rumiaciones excesivas, y a la severa autocrítica con la que se juzga. El perfeccionismo socialmente prescrito y el orientado a los demás son las dimensiones interpersonales; el primero hace referencia a las demandas que la persona perfeccionista percibe que los demás le imponen, y el desempeño que 
piensa que los demás esperan de él; en cambio, la última dimensión involucra las exigencias y críticas que el perfeccionista dirige a los demás, esperando de ellos desempeños perfectos.

El estilo cognitivo de los perfeccionistas insanos se caracteriza por la catastrofización, la sobregeneralización, la personalización, el pensamiento todo o nada y la atención selectiva centrada en el fracaso (Hewitt \& Flett, 1991). En el ámbito relacional, los perfeccionistas se vinculan con cierta hostilidad (su estilo comunicacional puede ser agresivo, son muy exigentes y críticos), al tiempo que tienen una gran sensibilidad interpersonal (basan su autoestima en la aceptación de los demás, necesitan de su aprobación y admiración). Por esto, suelen padecer diversos problemas en sus interacciones que, a su vez, pueden llevarlos a experimentar desconexión objetiva y subjetiva (Hewitt, Flett, Sherry, \& Caelian, 2006). Este estilo cognitivo y relacional junto a la necesidad de autocontrol, la autoevaluación constante y el miedo a fallar son los responsables del mantenimiento del perfeccionismo insano (De Rosa, 2012). Así también, explican el rol que el perfeccionismo puede cumplir en el surgimiento de desórdenes como ansiedad, depresión, trastornos alimentarios y deterioro de la salud física en general (Elizathe, Murawski, Custodio, \& Rutsztein, 2012; Oros, 2005; Sherry, Mackinnon \& Gautreau, 2016). Para una revisión más exhaustiva del perfil cognitivo de los perfeccionistas y su impacto sobre la salud puede consultarse Chemisquy (2018).

\section{El perfeccionismo en la escuela}

Las investigaciones científicas muestran que el perfeccionismo insano se encuentra presente incluso desde la niñez y que deriva en dificultades muy similares a las de la población adulta: depresión, ansiedad, trastornos obsesivos compulsivos, trastornos alimentarios y otros problemas como insomnio, agresividad y acumulación compulsiva (Affrunti \& WoodruffBorden, 2014; García Fernández et al., 2016; Morris \& Lomax, 2014; Oros, 2005; Vicent, Inglés, Sanmartín, Gonzálvez, \& García-Fernández, 2017).

En la escuela, los niños perfeccionistas pueden ser identificados porque se quedan terminando las tareas en el aula mientras sus compañeros están en el recreo, prefieren realizar las actividades solos y no gustan de los trabajos grupales, pueden ser muy prolijos y ordenados tanto en la realización de sus trabajos como en los espacios que ocupan dentro del aula, y suelen demorar en entregar sus pruebas para comprobar una y otra vez que las han hecho "a la perfección". En general, se enojan consigo mismos cuando no obtienen la calificación más alta, y algunos lo manifiestan con llanto y con reticencia a entrar al aula después de haber recibido una nota por debajo de lo anhelado. Pueden autoinculparse o culpar a los demás por sus errores, no suelen considerarse satisfechos, aunque obtengan buenos resultados, y pierden tanto tiempo 
en organizar un proyecto para que salga perfecto que dilatan iniciar la actividad (Kotman \& Ashby, 2000; Nugent, 2000; Rimm, 2007).

Los niños perfeccionistas perciben una amplia discrepancia entre los logros y las metas autoimpuestas, confían muy poco en sus capacidades para afrontar los problemas, temen fracasar en sus tareas y evaluaciones, y su rendimiento se empobrece, al igual que la satisfacción obtenida por sus propias calificaciones (Accordino, Accordino, \& Slaney, 2000; Bong, Hwang, Noh, \& Kim, 2014; Nounopoulos, Ashby, \& Gilman, 2006, Stoeber \& Rambow, 2007). Por lo tanto, es posible esperar que los niños con perfeccionismo insano experimenten más ansiedad ante tareas nuevas y que evalúen de manera negativa sus resultados, siempre pensando que podrían haberlo hecho mejor y que deberían ser mejores que los demás (DiBartolo \& Varner, 2012; Schruder, Curwen, \& Sharpe, 2014).

Si bien las manifestaciones perfeccionistas pueden evidenciarse simultáneamente en distintos ámbitos de actuación (académico, deportivo, social, etc.), es más frecuente que el perfeccionismo esté orientado a algunos dominios más que a otros (Dunn, Gotwals, \& Causgrove Dunn, 2005; McArdle, 2009; Stoeber \& Stoeber, 2009). Así, mientras algunos niños son más perfeccionistas con su desempeño académico, otros pueden serlo con relación a su rendimiento deportivo o a su apariencia física.

Con respecto al rendimiento deportivo, el perfeccionismo es fácilmente identificable en las clases de educación física o cuando los niños juegan en el recreo, siendo los perfeccionistas más proclives a enojarse y frustrarse cuando pierden en los juegos o cuando las cosas no salen tan perfectas como quisieran. Esta tendencia también se ha observado en adolescentes perfeccionistas involucrados en actividades deportivas de competición (Dunn, Gotwals, Dunn, \& Syrotuik, 2006).

En el área de las relaciones interpersonales, los niños perfeccionistas pueden estar mayormente aislados o sufrir acoso escolar (Roxborough et al., 2012), pueden evitar participar en actividades en las que no creen poder desempeñarse a la perfección (Gilman, Adams, \& Nounopoulos, 2010), o ser críticos con otros y exigirles que hagan todo perfecto, prefiriendo rodearse de los que juzgan como más aptos o inteligentes. Algunos niños pueden esforzarse por presentar una fachada de excelencia, con la cual se muestran como perfectos al tiempo que evitan hablar de sus imperfecciones o involucrarse en actividades donde hay riesgo de fallar (Flett \& Hewitt, 2013). Las dificultades de los niños perfeccionistas desadaptativos pueden manifestarse en forma de rechazo escolar (i.e. negativa de asistir a clases, concurrir y permanecer en el establecimiento escolar) (Gonzálvez Macia et al., 2015). Estos niños pueden dejar de asistir temporalmente a la escuela o presentar excusas para ello por temor a las 
evaluaciones, para evitar las situaciones sociales que les resultan aversivas debido a su sensibilidad interpersonal, o porque experimentan mucha ansiedad al separarse de sus cuidadores (Gonzálvez, Sanmartín, García-Fernández, Inglés, \& Vicent, 2016).

A pesar de las fuertes implicaciones que puede tener el perfeccionismo en el funcionamiento psicológico durante la edad escolar, siguen siendo escasas las investigaciones que abordan esta problemática y profundizan su comprensión como paso previo al desarrollo de estrategias de intervención. Algunos interrogantes que permanecen sin respuesta, al menos en el contexto latinoamericano, tienen que ver con la prevalencia de las manifestaciones perfeccionistas que exhiben los niños entre los 9 y los 12 años de edad. ¿Qué porcentaje de niños muestra valores elevados de perfeccionismo insano? Durante esta etapa del ciclo vital ¿predomina el perfeccionismo autorientado, el socialmente prescrito o el orientado a los demás? ¿Existen diferencias en las manifestaciones perfeccionistas según el género, el grado escolar, y el contexto en el que se desenvuelven los menores?

Investigaciones previas con población adulta canadiense y población infantil española han informado cierta preponderancia de la dimensión autorientada por sobre los aspectos interpersonales (Hewitt \& Flett, 1991; Lozano Fernández, García Cueto, Martín Vázquez, \& Lozano González, 2012), sin embargo, se requieren más evidencias para sustentar estos hallazgos y generar hipótesis.

Los resultados con respecto al género son divergentes. Algunos estudios realizados con niños y adolescentes han reportado valores más elevados de perfeccionismo insano en las mujeres. Entre estos trabajos se encuentra el de Uz Bas (2011) quien estudió una muestra de niños y niñas en Turquía y encontró que las mujeres puntuaron más elevado en algunas dimensiones de perfeccionismo como la sensibilidad a los errores, la autoestima contingente y la compulsión, pero con un tamaño de efecto bajo. Otros estudios han concluido que no hay diferencias entre varones y mujeres (Hewitt et al., 2002; Roselló \& Maysonet, 2002; Vuyk, 2015), mientras que una tercera franja de investigaciones ha encontrado que estas diferencias se muestran en favor de los hombres (Elizathe et al., 2012; Lozano Fernández et al., 2012; Serppe, Chemisquy, \& Oros, 2016; Vicent, Aparicio-Flores et al., 2017). En esta última línea también se encuadran los resultados de Pamies y Quiles (2014), aunque con un tamaño de efecto bajo. Hay también investigaciones con adolescentes superdotados que indican que ciertas dimensiones del perfeccionismo prevalecen en las mujeres (i.e. la preocupación excesiva por el orden y la precisión), mientras que otras sobresalen en los varones (i.e. la percepción de presión externa en base a altas expectativas parentales) (Siegle \& Schuler, 2000; Sondergeld, Schultz, \& Glover, 2007). 
Con relación al grado académico los estudios son mucho más limitados. Lozano Fernández et al. (2012) observaron en una muestra de niños que los niveles de perfeccionismo disminuían conforme los alumnos avanzaban en su escolaridad. Por su parte, Vuyk (2015) investigó una muestra de adolescentes de entre 13 y 19 años, sin encontrar diferencias significativas en función del grado académico. Estos últimos resultados se replicaron en el estudio de Vicent y sus colaboradores (2017), quienes concluyen que el perfeccionismo puede fluctuar ligeramente con la edad pero en definitiva debe considerarse como una variable estable. Poder contar con una caracterización local y precisa del perfeccionismo en edad escolar y de su relación con variables sociodemográficas permitirá desarrollar estrategias de intervención psicológicas y educativas basadas en la evidencia y orientadas a las necesidades reales de los destinatarios.

Así, el objetivo de este trabajo es describir las manifestaciones intra e interpersonales del perfeccionismo infantil disfuncional, identificando su prevalencia en función del género, el grado académico, el tipo de gestión escolar (pública-privada), y el lugar de procedencia de un grupo de niños que asisten regularmente a la escuela, con vistas a utilizar esta información para el posterior desarrollo de programas de prevención y tratamiento.

\section{Metodología}

\section{Tipo de estudio}

Se realizó una investigación empírica, cuantitativa, descriptiva, de corte transversal.

\section{Participantes}

La muestra estuvo compuesta por 1067 niños y niñas argentinos (44\% de varones), de entre 9 y 12 años de edad $(M=10.26 ; D E=1.01)$. El $37 \%$ de los encuestados asistía a establecimientos educativos de gestión pública, y el 63\% asistía a instituciones de gestión privada. De ellos, el $30.3 \%$ pertenecían al $4^{\circ}$ grado $(n=321)$, el $30.8 \%$ al $5^{\circ}$ grado $(n=326)$, el $37.3 \%$ al $6^{\circ}$ grado $(n=395)$ y el $1.5 \%$ restante al $7^{\circ}$ grado $(n=16)$. La elección de las escuelas se realizó por medio de un muestreo no probabilístico intencional, según las posibilidades de acceso a las instituciones. La delimitación por edad y curso se debió a que las pruebas de autorreporte utilizadas en la fase de evaluación fueron diseñadas especialmente para este rango etario.

El estudio se realizó en dos provincias argentinas: Misiones, que pertenece a la región nordeste $(n=636)$, y Entre Ríos, que forma parte de la región pampeana $(n=431)$. La provincia de Misiones, por estar ubicada en una de las zonas más pobres del país (Bolsi, Paolasso, \& 
Longhi, 2006), presenta mayores índices de necesidades básicas insatisfechas y analfabetismo que la provincia de Entre Ríos (INDEC, 2018; Ministerio de Salud, Organización Panamericana de la Salud, \& Organización Mundial de la Salud, 2017).

\section{Instrumentos}

Escala de Perfeccionismo Infantil (Oros, 2003). Esta escala de autoinforme evalúa el perfeccionismo autoorientado. Está constituida por 16 ítems tricotómicos que evalúan dos dimensiones del constructo: (a) Autodemandas (alpha $=.82$ ), que refleja las autoimposiciones y los estándares de los niños (ej. "Tengo que ser el mejor alumno", "Debo ganar siempre”); y (b) Reacciones ante el Fracaso (alpha $=.70)$, que mide las emociones y actitudes que aparecen ante los fallos y el incumplimiento de las metas (ej. "Me cuesta perdonarme cuando me equivoco", "Me siento culpable cuando cometo algún error") (Alpha de la escala completa = $.83)$.

Escala de Perfeccionismo Social Infantil (Oros, Serppe, Chemisquy, \& Ventura-León, inédito). Este instrumento evalúa las dos dimensiones interpersonales del perfeccionismo: (a) Perfeccionismo orientado a otros $(\omega=.83)$, que incluye 7 ítems sobre las exigencias y expectativas para con otros (ej. "Si le pido a alguien que haga algo, espero que lo haga perfecto"), y (b) Perfeccionismo socialmente prescrito $(\omega=.84)$ que consta de 9 ítems sobre las demandas percibidas como provenientes del medio externo (ej. "Mis padres quieren que sea perfecto en todo") (Omega general de la escala $=.89$ ).

\section{Procedimiento de recolección de datos}

Se solicitó permiso a los directivos de cada institución escolar por medio de una nota en la que se explicaron los objetivos de la investigación. Una vez logrado el acceso a las aulas se entregaron consentimientos informados por escrito a cada uno de los niños para que los padres y/o tutores aprobaran su participación, con el fin de mantener los resguardos éticos necesarios. La administración de los instrumentos estuvo a cargo de miembros del equipo de investigación capacitados para tal fin, y se realizó de manera grupal durante horas de clase, en el aula, en presencia de los docentes de cada grado.

\section{Procedimiento estadístico}

Los datos fueron procesados con el programa SPSS, versión 20. En primer lugar, se reemplazaron los datos perdidos con el método de imputación del valor de la media individual, que consiste en la sustitución de cada valor perdido por la media que obtuvo el niño en la sub- 
escala (Shrive, Stuart, Quan, \& Ghali, 2006). Seguidamente, se observó la normalidad univariada de los datos a través de: (a) las puntuaciones estándar, donde puntajes mayores a $z$ = 3.29 se consideraron atípicos; (b) los gráficos de cajas o boxplots, en los que se observó la presencia de outliers; y (c) los valores de asimetría y curtosis, considerando como excelentes a los valores en el rango entre $-1 \mathrm{y}+1$, y aceptables los valores entre $-2 \mathrm{y}+2$ (George \& Mallery, 2010). En tercer lugar, se calcularon los valores medios y desvíos estándar de las distintas dimensiones evaluadas y de los ítems que las componían, a fin de determinar la prevalencia de las principales manifestaciones del perfeccionismo en niños de edad escolar. Para este análisis las medias estuvieron ponderadas por la cantidad de elementos que incluía cada dimensión con el fin de lograr valores comparables entre sí. De esta manera, los valores de las tres subescalas (perfeccionismo autorientado, orientado a otros y socialmente prescrito) oscilaron entre un valor mínimo de 1 y un máximo de 3. Los demás análisis de medias se realizaron sobre los valores originales. En cuarto lugar, se ejecutaron las pruebas no paramétricas U de MannWhitney y Kruskal Wallis para analizar las diferencias en los puntajes de las dimensiones del perfeccionismo según el género, la gestión escolar, el lugar de procedencia de los menores y el grado académico. Para este último análisis la muestra fue agrupada en tres segmentos: 4to (30.3\%), 5to (30.8\%) y 6to-7mo grados $(38.8 \%)$ para lograr una distribución más equitativa de los niños. La selección de las pruebas no paramétricas para la comparación de medias se debió al incumplimiento del supuesto de normalidad, según la prueba de Levene.

\section{Resultados}

El porcentaje de datos perdidos correspondiente a participantes que no contestaron algún ítem de la escala no superó el $4.1 \%$ para cada elemento, siendo el ítem $\mathrm{N}^{\mathrm{o}} 5$ de la dimensión Socialmente Prescrita ("Mis padres me exigen tanto que nunca los puedo dejar contentos") el que mayor cantidad de respuestas vacías obtuvo. Los valores perdidos debido a la deserción de los participantes no alcanzaron el 5\%, por lo que no se realizó imputación de estos datos.

Tras el análisis de atípicos se identificó un total de 26 casos que cumplían esta condición, los cuales fueron cuidadosamente estudiados para encontrar las posibles causas. La mayoría de estos casos respondían a puntajes muy elevados pero sin indicios de errores en la medición. El análisis de curtosis y asimetría de la muestra completa arrojó resultados excelentes para la escala de perfeccionismo autorientado ( $A s=0.34 ; C u=-0.17)$ y para la subescala de perfeccionismo socialmente prescrito $(A s=0.63 ; C u=-0.33)$, y resultados considerados poco a no aceptables para la escala de perfeccionismo orientado a los otros $(A s=1.62 ; C u=2.63)$. 
Teniendo en cuenta este análisis y considerando que el objetivo de este trabajo es describir la prevalencia del perfeccionismo infantil, se procedió a analizar la muestra completa ya que los casos atípicos podrían estar representando a niños con elevados niveles de perfeccionismo, que no deberían quedar fuera de la exploración.

Tomando en consideración el rango posible de puntuaciones ponderadas, que va desde 1 a 3 puntos, se puede estimar que, en general, los niños presentan valores moderadamente bajos y bajos de perfeccionismo, ya que las medias obtenidas oscilaron entre 1.32 y 1.82 puntos. Dado que existe un baremo nacional que aporta criterios de referencia para interpretar los valores de perfeccionismo autorientado, fue posible identificar la proporción de niños de esta muestra que se encuentra en mayor condición de riesgo psicológico respecto de esta dimensión. A partir de los datos recabados se aprecia que el 52.70\% de los varones presentan valores bajos de perfeccionismo autorientado, en tanto que el $22.40 \%$ presenta valores moderados y el $23.10 \%$ valores de riesgo. Con relación a las niñas, el $60.1 \%$ obtuvo valores bajos, el $20.7 \%$ alcanzó valores moderados, y el 19.2\% presentó valores de riesgo.

Por otra parte, los resultados indicaron que en esta muestra de niños prevalece el perfeccionismo autorientado $(M=1.82 ; D E=0.38)$ por sobre el perfeccionismo socialmente prescrito $(M=1.62 ; D E=0.48)$ y el perfeccionismo orientado a otros $(M=1.32 ; D E=0.37)$. Además, el análisis de las correlaciones bivariadas entre las distintas dimensiones del perfeccionismo señala una asociación importante entre todas ellas: (a) autorientado y socialmente prescrito: $r=.59(p<.001)$; (b) orientado a otros y a uno mismo: $r=.50(p<.001)$; y (c) socialmente prescrito y orientado a otros: $r=.46(p<.001)$.

Un análisis más pormenorizado reveló además que dentro del perfeccionismo autorientado, las dos demandas más sobresalientes serían: "Tengo que ser el mejor alumno" y "No puedo cometer errores", y las reacciones negativas más frecuentes serían: "Pienso mucho en las equivocaciones que tuve" y "Me siento culpable cuando cometo un error". Por otra parte, las dos exigencias sociales percibidas con mayor intensidad serían: "Mis maestros esperan que haga mi tarea perfecta" y "Siento que no puedo cumplir con todo lo que mis padres esperan de mí”. Por último, las manifestaciones perfeccionistas más habitualmente experimentadas hacia otros serían: "Me molestan los chicos que no entienden las cosas rápido" y "Trato de juntarme con los más inteligentes". (Ver Tabla 1).

Tabla 1

Valores promedio y desvíos estándar para cada ítem de perfeccionismo 


\begin{tabular}{lcc}
\hline Ítems de cada escala & Media & DE \\
\hline Autodemandas & & \\
Necesito ser el mejor & 1.88 & 0.76 \\
Tengo que ser el mejor alumno & $\mathbf{2 . 0 1}$ & $\mathbf{0 . 7 9}$ \\
Debo ganar siempre & 1.56 & 0.70 \\
No puedo cometer errores & $\mathbf{1 . 9 5}$ & $\mathbf{0 . 8 1}$ \\
Mis trabajos tienen que ser mejores que los demás & 1.53 & 0.71 \\
No debo perder cuando juego con mis amigos & 1.55 & 0.73 \\
Debo ser el mejor de la clase & 1.72 & 0.78 \\
Debo ser el primero en terminar las tareas escolares & 1.70 & 0.77
\end{tabular}

Reacciones ante el fracaso

Cuando pierdo me siento mal

$1.85 \quad 0.71$

Pienso mucho en las equivocaciones que tuve

$2.40 \quad 0.70$

Me siento muy mal cuando pierdo en algún juego o deporte

$1.61 \quad 0.72$

Me cuesta perdonarme cuando me equivoco

$1.91 \quad 0.82$

Me enojo cuando no logro lo que quiero

$1.75 \quad 0.74$

Me critico mucho a mi mismo

$1.65 \quad 0.77$

Me siento culpable cuando cometo algún error

$2.38 \quad 0.72$

Me insulto cada vez que cometo un error

$1.57 \quad 0.76$

Perfeccionismo socialmente prescrito

Mi familia quiere que yo sea perfecto

$1.61 \quad 0.77$

Mis padres me piden que haga todo perfecto

$1.65 \quad 0.77$

Mis maestros esperan que haga mi tarea perfecta

$2.02 \quad 0.85$

Mis padres quieren que sea el mejor en todo

$1.49 \quad 0.71$

Mis padres me exigen tanto que nunca los puedo dejar contentos

$1.42 \quad 0.66$

Siento que no puedo cumplir con todo lo que mis padres esperan de mí

$1.87 \quad 0.76$

Mis maestros no aceptan que yo cometa errores

$1.68 \quad 0.80$

En mi casa me castigan (me retan) si no hago las cosas perfectas

$1.44 \quad 0.68$

Mis padres critican todo lo que hago (encuentran errores en todo lo que

$1.40 \quad 0.66$ hago)

\section{Perfeccionismo orientado a otros}

Me enojo con mis amigos cuando no quieren sacar notas altas

$1.30 \quad 0.61$

Me enojo con las personas que no hacen bien sus trabajos

$1.27 \quad 0.57$

Me molestan los chicos que no entienden las cosas rápido

$1.53 \quad 0.71$

Me da rabia que los demás no sean perfectos

$1.17 \quad 0.48$

Trato de juntarme con los más inteligentes

$1.51 \quad 0.52$ 
Me molesta que mi mejor amigo o amiga no quiera ser perfecto

Me caen mal las personas que no son muy inteligentes
$1.24 \quad 0.56$

$1.21 \quad 0.51$

Nota. Los dos ítems de cada dimensión con valores medios más elevados están señalados en negrita.

Los resultados mostraron además que estas manifestaciones varían en función del género. Se observaron diferencias significativas entre niñas y varones en el perfeccionismo autorientado $(U=108059.5 ; p<.001)$, el perfeccionismo socialmente prescrito $(U=99191.5$; $p<.001)$ y el orientado a los otros $(U=105145 ; p=.001)$. En el caso del perfeccionismo autorientado, las diferencias se apreciaron tanto en la subdimensión Autodemandas $(U=$ $104273.0 ; p<.001)$ como en la de Reacciones ante el fracaso $(U=120604.0 ; p=.005)$. En todos los casos los varones puntuaron más alto que las niñas (ver Tabla 2).

Tabla 2

Valores de perfeccionismo en función del género

\begin{tabular}{lcccc}
\hline & \multicolumn{2}{c}{ Masculino } & \multicolumn{2}{c}{ Femenino } \\
\cline { 2 - 5 } & $\boldsymbol{R M}$ & $\boldsymbol{S R}$ & $\boldsymbol{R M}$ & $\boldsymbol{S R}$ \\
\hline Perf. autorientado & 578.58 & 265566.50 & 477.53 & 278879.50 \\
$\quad$ Autodemandas & 586.83 & 269353.00 & 471.05 & 275093.00 \\
$\quad$ Reacciones & 551.25 & 253022.00 & 499.01 & 291424.00 \\
Perf. socialmente prescrito & 586.96 & 268236.5 & 460.51 & 264791.5 \\
Perf. orientado a otros & 566.9 & 257374 & 470.14 & 268451 \\
\hline
\end{tabular}

Nota. $R M=$ Rango medio; $S R=$ Suma de rangos.

Con el objeto de arrojar mayor luz sobre el tema, y debido a las divergencias observadas en los resultados de estudios anteriores, se decidió realizar un análisis más detallado de las diferencias por género encontradas en este trabajo. Se estudió puntualmente en qué ítems de las dimensiones del perfeccionismo difieren más los niños de las niñas. Con respecto a las autoexigencias, las mayores diferencias se observaron, a favor de los varones, en los siguientes tres ítems: "Necesito ser el mejor" $(U=107663 ; p<.001)$, "Debo ganar siempre" $(U=110551$; $p<.001) \mathrm{y}$ "No debo perder cuando juego con mis amigos" $(U=110829 ; p<.001)$. Por el contrario, no se observaron diferencias significativas entre varones y niñas respecto a los ítems: "Mis trabajos deben ser mejores que los demás" $(U=126219 ; p=.065)$ y "Debo ser el primero en terminar las tareas escolares" $(U=127711 ; p=.155)$. Respecto de las reacciones ante el fracaso, las diferencias entre géneros se comprobaron en los ítems: "Me cuesta perdonarme cuando me equivoco" $(U=124696.5 ; p=.045)$, "Me insulto cada vez que cometo un error" $(U$ 
$=124360 ; p=.026), \mathrm{y}$ "Me enojo cuando no logro lo que quiero" $(U=117156.5 ; p<.001)$, siendo este último el que reflejó las diferencias más marcadas.

En cuanto al perfeccionismo socialmente prescrito las diferencias más marcadas en favor de los varones se encontraron en los ítems: "Mi familia quiere que yo sea perfecto" ( $U=107676.5 ; p$ $<.001)$, "Mis padres piden que haga todo perfecto" $(U=106921 ; p<.001) \mathrm{y}$ "Mis padres me exigen tanto que nunca los puedo dejar contentos" $(U=110498 ; p<.001)$.

Por último, con respecto a la subescala de perfeccionismo orientado a los otros las mayores diferencias se observaron en los siguientes tres ítems: "Trato de juntarme con los más inteligentes $(U=98926 ; p<.001)$, "Me caen mal las personas que no son muy inteligentes" ( $U$ $=116781 ; p<.001), \mathrm{y}$ "Me molesta que mi mejor amigo o amiga no quiera ser perfecto" ( $U=$ 120937; $p=.005)$, aunque el ítem "Me da rabia que los demás no sean perfectos" ( $U=$ $121182.5 ; p=.001)$ también se destacó con una diferencia de promedios muy cercana a la del ítem anterior.

Los promedios de cada ítem en las submuestras de niñas y varones se pueden apreciar en la Tabla 3.

Tabla 3

Promedios y diferencias de los ítems de cada dimensión entre niñas y varones

\begin{tabular}{lccc}
\hline & \multicolumn{2}{c}{ Rangos promedio } & \multirow{2}{*}{ Dif Rp } \\
\cline { 2 - 3 } & Varones & Niñas & \\
\cline { 2 - 3 } Autodemandas & & & \\
Necesito ser el mejor & $\mathbf{5 7 9 . 4 4}$ & $\mathbf{4 7 6 . 8 5}$ & $\mathbf{1 0 2 . 5 9 * * *}$ \\
Tengo que ser el mejor alumno & 556.93 & 494.55 & $62.38^{* * * *}$ \\
Debo ganar siempre & $\mathbf{5 7 3 . 1 5}$ & $\mathbf{4 8 1 . 8 0}$ & $\mathbf{9 1 . 3 5 * * *}$ \\
No puedo cometer errores & 556.85 & 494.61 & $62.24^{* * *}$ \\
Mis trabajos tienen que ser mejores que los demás & 539.01 & 508.63 & 30.38 \\
No debo perder cuando juego con mis amigos & $\mathbf{5 7 2 . 5 4}$ & $\mathbf{4 8 2 . 2 8}$ & $\mathbf{9 0 . 2 6} * * *$ \\
Debo ser el mejor de la clase & 564.31 & 488.75 & $75.56^{* * * *}$ \\
Debo ser el primero en terminar las tareas & 535.76 & 511.18 & 24.58 \\
escolares & & & \\
Reacciones ante el fracaso & & & \\
Cuando pierdo me siento mal & & & \\
Pienso mucho en las equivocaciones que tuve & 528.31 & 516.14 & 12.17 \\
Me siento muy mal cuando pierdo en algún juego o & 536.84 & 509.42 & 27.42 \\
\hline
\end{tabular}


deporte

Me cuesta perdonarme cuando me equivoco

Me enojo cuando no logro lo que quiero

Me critico mucho a mi mismo

Me siento culpable cuando cometo algún error

Me insulto cada vez que cometo un error

Perfeccionismo socialmente prescrito

\section{Mi familia quiere que yo sea perfecto}

Mis padres me piden que haga todo perfecto

Mis maestros esperan que haga mi tarea perfecta

Mis padres quieren que sea el mejor en todo

Mis padres me exigen tanto que nunca los

puedo dejar contentos

Siento que no puedo cumplir con todo lo que mis

padres esperan de mi

Mis maestros no aceptan que yo cometa errores

En mi casa me castigan (me retan) si no hago las cosas perfectas

Mis padres critican todo lo que hago (encuentran errores en todo lo que hago)

\section{Perfeccionismo orientado a otros}

Me enojo con mis amigos cuando no quieren sacar notas altas

Me enojo con las personas que no hacen bien sus trabajos

Me molestan los chicos que no entienden las cosas rápido

Me da rabia que los demás no sean perfectos

Trato de juntarme con los más inteligentes

Me molesta que mi mejor amigo o amiga no quiera ser perfecto

Me caen mal las personas que no son muy inteligentes

$\begin{array}{lll}\mathbf{5 4 1 . 3 3} & \mathbf{5 0 5 . 8 9} & \mathbf{3 5 . 4 4} * \\ \mathbf{5 5 7 . 7 6} & \mathbf{4 9 2 . 9 5} & \mathbf{6 4 . 8 1} * * * \\ 535.59 & 510.40 & 25.19 \\ 533.88 & 511.75 & 22.33 \\ \mathbf{5 4 2 . 0 6} & \mathbf{5 0 5 . 3 1} & \mathbf{3 6 . 7 5} *\end{array}$

$568.38 \quad 475.26 \quad 93.12 * * *$

$570.04 \quad 473.95 \quad 96.09 * * *$

$554.26 \quad 486.49 \quad 67.77 * * *$

$554.80 \quad 486.06 \quad 68.74 * * *$

$562.21 \quad 480.17 \quad 82.04 * * *$

$532.47 \quad 503.81 \quad 28.66$

$550.33 \quad 489.61 \quad 60.72^{* * *}$

$550.86 \quad 489.19 \quad 61.67 * * *$

$557.63 \quad 483.81 \quad 73.82 * * *$

$\begin{array}{lll}518.85 & 508.35 & 10.05\end{array}$

$\begin{array}{lll}525.33 & 503.20 \quad 22.13\end{array}$

$523.31 \quad 504.80 \quad 18.51$

$531.58 \quad 498.23 \quad 33.35^{* *}$

$580.60 \quad 459.25 \quad 121.35 * * *$

$\begin{array}{lll}532.12 & 497.80 & 34.32 * *\end{array}$

541.27 $490.52 \quad 50.75 * * *$

$\overline{\text { Nota. } * p<.05 ; * * p<.01 ; * * * p<.001 . \text { Dif Rp: Diferencia de Rangos promedio. Se han señalado con }}$ negrita los ítems con mayor Dif Rp. 
En otro orden de análisis, se observó que el grado al que asisten los niños también mostró ser una variable relevante, tanto para el perfeccionismo autorientado (Kruskal Wallis $(2)=18.6 ; p<.001)$ como para el socialmente prescrito (Kruskal Wallis $(2)=21.41 ; p<.001)$. Para el caso del perfeccionismo autorientado, las diferencias entre grados fue más significativa para la dimensión de Autodemandas (Kruskal Wallis $(2)=21.58 ; p<.001$ ) que para las Reacciones frente al fracaso (Kruskal Wallis $(2)=7.69 ; p=.021$ ). Por su parte, no se observaron diferencias entre los distintos grados en relación al perfeccionismo orientado a otros (Kruskal Wallis $(2)=4.28 ; p=.118)$. Las pruebas $\mathrm{U}$ de Mann-Whitney indicaron que las diferencias se encuentran principalmente entre los niños de cuarto grado respecto de sus compañeros del sexto y séptimo curso (Perfeccionismo autorientado: $U=54459 ; p<.001$; Autodemandas: $U=53646$; $p<.001$; Reacciones frente al fracaso: $U=58682 ; p=.006$; Perfeccionismo socialmente prescrito: $U=52361 ; p<.001)$. También se encontraron diferencias para la dimensión Autorientada entre los niños de cuarto y quinto grado $(U=46945 ; p=.014)$, y sólo para la subdimensión de Autodemandas para los alumnos de quinto en relación a sus congéneres de sexto y séptimo grado $(U=60258 ; p=.006)$. Los niños de 6to-7mo grados presentan menor perfeccionismo intrapersonal y socialmente prescrito que los demás niños (ver Tabla 4).

Tabla 4

Comparaciones múltiples del perfeccionismo autorientado y el socialmente prescrito según el grado académico

\begin{tabular}{|c|c|c|c|}
\hline & 4to grado & 5to grado & 6to-7to grados \\
\hline & $R M$ & $R M$ & $R M$ \\
\hline Perf. autorientado & 588.10 & 532.20 & 489.63 \\
\hline Autodemandas & 587.24 & 543.43 & 482.63 \\
\hline Reacciones frente al fracaso & 570.50 & 525.96 & 508.23 \\
\hline Perf. socialmente prescrito & 575.79 & 543.64 & $\begin{array}{c}474.63 \\
\end{array}$ \\
\hline Perf. orientado a otros & 550.59 & 504.99 & 514.64 \\
\hline
\end{tabular}

$\overline{\text { Nota. Los corchetes indican entre qué grupos se presentan las diferencias significativas al nivel de: }}$ $p<.05 ;-p<.001$

Para revisar el impacto del tipo de escuela (privada-pública) se realizó la prueba $U$ de 
Mann-Whitney encontrando resultados significativos tanto para el perfeccionismo autorientado $(U=118764.5 ; p<.040)$, como para el socialmente prescrito $(U=106898.5 ; p<.001)$ y el orientado a los otros $(U=103290 ; p<.001)$. Las diferencias en el perfeccionismo autorientado se presentaron únicamente en la subdimensión de Autodemandas $(U=115908.5 ; p<.007)$. En todos estos casos las puntuaciones obtenidas por los niños de escuelas estatales fueron mayores a las obtenidas por los niños de escuelas privadas (ver Tabla 5).

Tabla 5

Valores de perfeccionismo según el tipo de gestión escolar

\begin{tabular}{lcccc}
\hline & \multicolumn{2}{c}{ Pública } & \multicolumn{2}{c}{ Privada } \\
\cline { 2 - 5 } & $\boldsymbol{R M}$ & $\boldsymbol{S R}$ & $\boldsymbol{R M}$ & $\boldsymbol{S R}$ \\
\cline { 2 - 5 } Perf. autorientado (escala general) & 560.15 & 206693.5 & 519.39 & 362017.5 \\
$\quad$ Autodemandas & 568.88 & 209918.5 & 515.56 & 359859.5 \\
$\quad$ Reacciones ante el fracaso & 538.26 & 198618.5 & 530.98 & 370092.5 \\
Perf. socialmente prescrito & 579.72 & 212758.5 & 499.60 & 343226.5 \\
Perf. orientado a otros & 580.45 & 210705 & 493.23 & 336876 \\
\hline
\end{tabular}

Nota. $R M=$ Rango medio; $S R=$ Suma de rangos.

Finalmente, se analizaron las diferencias en los valores de perfeccionismo infantil en relación a la provincia de procedencia por medio de la prueba de $U$ de Mann-Whitney. Se encontraron diferencias significativas para las autodemandas $(U=122252 ; p=.003)$, el perfeccionismo socialmente prescrito $(U=118008$; $p=.001)$ y el perfeccionismo orientado a los otros $(U=115580.5 ; p=.001)$. Los datos indican valores más elevados en estas escalas de perfeccionismo para los niños de la provincia de Misiones (ver Tabla 6).

Tabla 6

Valores de perfeccionismo según la provincia de procedencia

\begin{tabular}{lcccc}
\cline { 2 - 5 } & \multicolumn{2}{c}{ Misiones } & \multicolumn{2}{c}{ Entre Ríos } \\
\cline { 2 - 5 } & $\boldsymbol{R M}$ & $\boldsymbol{S R}$ & $\boldsymbol{R M}$ & $\boldsymbol{S R}$ \\
\hline Perf. autorientado & 548.05 & 548.05 & 511.98 & 220149.5 \\
$\quad$ Autodemandas & 557.08 & 354861 & 499.81 & 214917 \\
$\quad$ Reacciones ante el fracaso & 533.54 & 339330.5 & 533.44 & 229380.5 \\
Perf. socialmente prescrito & 551.98 & 348301 & 490.98 & 207684 \\
Perf. orientado a otros & 548.25 & 344847.5 & 486.17 & 202733.5 \\
\hline
\end{tabular}

Nota. $R M=$ Rango medio; $S R=$ Suma de rangos. 


\section{Discusión}

El objetivo del presente estudio fue identificar la prevalencia de las principales manifestaciones del perfeccionismo infantil en niños escolarizados. Los resultados mostraron valores de perfeccionismo entre bajos y moderados, y se identificaron algunos casos en condición de riesgo. Al trabajar con población no clínica era esperable obtener puntajes bajos, pero la presencia de los casos en riesgo indica la necesidad de profundizar en los estudios de intervención preventiva en el ámbito escolar.

Por otra parte, los resultados mostraron correlaciones significativas entre las tres facetas del perfeccionismo, lo que indicaría que los aspectos intrapersonales co-existen con los interpersonales en la definición del perfil perfeccionista infantil. Estos resultados se encuentran en línea con los de Hewitt y Flett (1991) en población adulta, quienes hallaron correlaciones que oscilaron entre $r=.25$ a .40; por su parte, Stoeber, Noland, Mawenu, Henderson, y Kent (2017) también encontraron correlaciones entre los tres tipos de perfeccionismo con valores fluctuantes entre .16 y .44 .

En cuanto a los análisis dimensionales, los datos indicaron que la manifestación más prominente en la muestra analizada es de naturaleza intrapersonal. Los niños se autoimponen excesivas aspiraciones de rendimiento, destacándose entre ellas las exigencias de ser el mejor alumno y no cometer errores. Por otro lado, las reacciones infantiles más habituales frente al incumplimiento de estas metas autoimpuestas fueron la autoinculpación y la rumiación. Entre un $19 \%$ y un $23 \%$ de los niños evaluados mostraron una tendencia extrema hacia esta dimensión del perfeccionismo (autorientada), lo cual podría considerarse una condición de riesgo para la salud mental.

A la experiencia intrapersonal le sigue en orden de importancia la percepción de que los padres y maestros esperan de ellos no menos que la perfección, lo cual implica no equivocarse ni cometer errores al momento de cumplir con una consigna o solicitud. A esta manifestación le sigue en el orden de importancia una actitud de impaciencia y rechazo hacia las personas que difieren del estándar que ellos mismos fijan como aceptable.

La prevalencia del perfeccionismo autorientado por encima de las dimensiones interpersonales también ha sido observada en otros estudios con niños (Lozano Fernández et al., 2012) y adultos (Hewitt \& Flett, 1991), lo que abona la idea de que las autoexigencias desmedidas constituyen uno de los rasgos prioritarios que caracterizan esta disfunción (Shafran, Cooper, \& Fairburn, 2002).

Es importante destacar que en la muestra general, la autoexigencia perfeccionista más 
marcada se observó en relación al desempeño escolar y no a las actividades lúdico-deportivas, que también son muy importantes en esta etapa. Si bien es deseable que los menores en edad escolar aspiren a ser buenos alumnos y organicen sus recursos y habilidades para cumplir con esta meta, los niños perfeccionistas confunden el deseo de ser mejores estudiantes con la obligación de serlo. Cambian las demandas preferenciales (me gustaría, quisiera, preferiría ser el mejor alumno) por demandas absolutistas, rígidas e irracionales (debo, tengo, estoy obligado a ser el mejor) (Ellis, 2002). Esta meta, que es vista por los niños como decisiva para lograr la aceptación y la aprobación de sí mismos, no es alcanzable en tanto la definición de "mejor alumno" siempre es perfectible. Por ende, los niños perfeccionistas se encuentran sumidos en un estado de perplejidad, angustia e insatisfacción permanente a pesar de los grandes esfuerzos que hacen para progresar (Egan, Wade, Shafran, \& Anthony, 2014).

Por otro lado, frente a la no consecución de esos estándares auto-establecidos, las reacciones más habituales manifiestan la tendencia a devaluarse a sí mismos (DiBartolo, Frost, Chang, LaSota, \& Grills, 2004) y a iniciar procesos de pensamientos negativos repetitivos (Macedo et al., 2015). Esto se ha observado en este estudio al verificar la presencia de puntajes elevados en los ítems que indagaban acerca de la culpa y la rumiación como reacción ante el fracaso. Cuando el estado afectivo consecuente a la percepción de fallos o equivocaciones en el desempeño y en la obtención de las metas autoimpuestas, se incrementa en frecuencia e intensidad puede derivar en un importante desajuste emocional caracterizado por la depresión y la ansiedad (Hewitt et al., 2002).

En este estudio también se encontró que las manifestaciones perfeccionistas varían según ciertas variables sociodemográficas. Por ejemplo, los varones presentaron puntajes más elevados en todos los tipos de perfeccionismo respecto de las mujeres. Estos resultados coinciden con los informados por Elizathe et al. (2012), Lozano Fernández et al. (2012) y Serppe et al. (2016) quienes también han reportado más autoexigencia en los varones en edad escolar. El análisis por ítems reveló que el mayor perfeccionismo en los varones afloraría especialmente en referencia a situaciones lúdicas, socio-recreativas o deportivas. Estos resultados pueden deberse entre otras causas, a que en general, los varones están marcados por expectativas tradicionales de género que exigen actitudes de mayor competencia, autosuficiencia, fuerza, vigor y velocidad. Según los estereotipos de rol, estas cualidades indicarían mayor virilidad y posibilidades de ser socialmente aceptados, condiciones que resultan especialmente deseables en la niñez tardía y la adolescencia (Adams \& Govender, 2008). Sin embargo, considerando lo que plantean Vicent y sus colaboradores (2017) las diferencias percibidas entre los géneros en el nivel de la medición no siempre representan una 
diferencia para la práctica clínica, y además existen otras variables que pueden modificar la relación género-perfeccionismo. Por otra parte, las niñas podrían aventajar a los varones en ciertos aspectos del perfeccionismo no examinados en este estudio (i.e. sensibilidad a los errores, compulsividad, pulcritud, apariencia física), por lo que sería prematuro afirmar que los varones son más perfeccionistas que las niñas. En todo caso, lo que puede concluirse es que el perfeccionismo se manifiesta diferencialmente en varones y niñas según los estereotipos sociales, las expectativas de género y las condiciones biológicas de base, y que su impacto sobre la salud de ambos también puede variar en virtud de numerosos factores.

En cuanto a las reacciones ante el fracaso, se observó que los varones puntúan más alto que las niñas en dos ítems que se aproximan a conductas externalizantes o agresivas ("Me enojo cuando no logro lo que quiero", "Me insulto cada vez que cometo un error"). Nuevamente, este hallazgo podría tener su explicación en los estereotipos de rol a merced de los cuales se ha observado que los varones suelen ser más reactivos y manifestar más conductas externalizantes en comparación a sus pares mujeres (Samaniego, 2004, 2008). De cualquier manera, estos datos deberían ser corroborados en próximos estudios para llegar a una explicación concluyente.

Con relación al perfeccionismo socialmente prescrito se observó que los varones perciben una exigencia parental más desmedida que las niñas para ser y hacer todo perfecto, demanda que naturalmente no alcanza a satisfacerse. Estos resultados coinciden con los reportados por Flett, Hewitt, Boucher, Davidson y Munro (2000) y sería interesante profundizarlos en próximas investigaciones para dilucidar si efectivamente se les exige más a los hijos varones o si se trata de una percepción subjetiva de estos últimos.

Finalmente, con relación a la subescala de perfeccionismo orientado a los otros se encontraron puntajes significativamente superiores para los varones en los ítems "Me da rabia que los demás no sean perfectos", "Me molesta que mi mejor amigo o amiga no quiera ser perfecto", "Me caen mal las personas que no son muy inteligentes" y "Trato de juntarme con los más inteligentes". Estos resultados indican que los varones, quienes se diferencian de las niñas por percibir mayor exigencia externa para ser nada menos que perfectos, repiten en sus relaciones con otros las mismas exigencias directas de perfección que perciben de sus padres. Esto ratificaría que ciertos aspectos del perfeccionismo infantil se adquieren por aprendizaje y observación de las figuras significativas, y por las relaciones que con ellas se establecen, además de las propias disposiciones temperamentales del niño (Barrow \& Moore, 1983; Blatt, 1995; Flett, Hewitt, Oliver, \& Macdonald, 2002; Ocampo 2008; Oros, Iuorno, \& Serppe, 2017).

Por otra parte, los resultados muestran una disminución del perfeccionismo autorientado y socialmente prescrito a medida que transcurre el progreso escolar durante el segundo ciclo 
(del cuarto al séptimo grado). Estos resultados también fueron observados por Lozano Fernández et al. (2012). Según estos autores, es posible que a medida que los niños crecen, las demandas familiares se vuelvan menos intrusivas y la autonomía infantil más desarrollada, lo que explicaría el descenso en los puntajes de perfeccionismo socialmente prescrito. Al mismo tiempo, y dado que estas dos dimensiones están muy relacionadas entre sí, la percepción de una menor presión social podría explicar la disminución del perfeccionismo intrapersonal. Aun así, un análisis adicional mostró que durante los dos últimos años de la escolarización primaria se sigue observando un porcentaje de entre el 17\% y el 14\% de niños en situación de riesgo por sus niveles elevados de perfeccionismo autorientado, lo que resulta coincidente con otros estudios que señalan que el perfeccionismo disfuncional puede mantenerse bastante estable a lo largo del tiempo más allá de algunas fluctuaciones menores (Vicent, Aparicio-Flores et al., 2017; Schruder et al., 2014). La relación entre el perfeccionismo y la edad requiere de nuevos estudios, especialmente longitudinales, para poder extraer conclusiones precisas.

Con respecto al tipo de gestión escolar y el lugar de procedencia, los resultados mostraron mayores puntuaciones de perfeccionismo para los alumnos de escuelas públicas y para los residentes en la provincia de Misiones. Debido a que no hay estudios previos que hayan analizado el perfeccionismo infantil a la luz de estas variables externas, no es sencillo establecer conclusiones al respecto. Con relación a las escuelas, las diferencias podrían deberse a que las instituciones privadas suelen caracterizarse por mejores climas laborales y mayores oportunidades de capacitación y formación continua de sus recursos humanos (Krüger \& Formichella, 2012), lo cual podría significar la presencia de docentes menos exhaustos y mejor preparados para identificar y atender a tiempo a niños con creencias, actitudes y comportamientos perfeccionistas.

Otra posible hipótesis podría orientar la explicación de estos resultados hacia el ambiente social del que proviene el niño, ya que hay mayor probabilidad de encontrar niños de clase socioeconómica baja en las escuelas públicas y en la región nordeste del país. Como ya ha sido mencionado, la provincia de Misiones se caracteriza por tener mayores índices de pobreza que la provincia de Entre Ríos, lo que podría significar una condición de riesgo psicosocial para el desarrollo afectivo y cognitivo de su población infantil. Existen abundantes evidencias científicas respecto al efecto nocivo de la vulnerabilidad asociada a la pobreza sobre el crecimiento, el desarrollo social, cognitivo, afectivo y comportamental, y sobre la salud física de los menores, especialmente cuando su presencia es persistente y se inicia en las primeras etapas de la vida (Richaud de Minzi, 2007). Si bien hasta el momento no se han encontrado estudios que relacionen directamente el nivel socio económico con el perfeccionismo, no hay 
que dejar de lado el impacto que tienen los factores ambientales en el desarrollo de esta disfunción cognitiva (Flett et al., 2002). Además, se ha comprobado que los adultos que ejercen sus funciones parentales en condiciones de pobreza relativa y pobreza extrema, suelen presentar, debido a los elevados niveles de estrés a los que están expuestos: altos índices de control patológico hacia los hijos, baja implicación afectiva, indiferencia y negligencia (Vargas Rubilar, 2015; Vargas Rubilar, Lemos, \& Richaud, 2017), comportamientos parentales que a su vez promueven el desarrollo del perfeccionismo infantil (Affrunti \& Woodruff-Borden, 2014; Oros, 2000; McArdle, 2009).

En vistas de estos resultados, sería importante continuar investigando esta línea para poder encontrar los factores sociales, culturales y familiares que están en juego en estas diferencias y así conocer mejor los factores de riesgo que aumentan la vulnerabilidad de los niños para desarrollar el perfeccionismo insano. La identificación de estos factores permitirá diseñar estrategias de intervención encaminadas a la reducción y la prevención de creencias perfeccionistas irracionales. Los padres, docentes y terapeutas pueden torcer el curso negativo de ciertos desórdenes si actúan tempranamente en la prevención y modificación de estos patrones rígidos de pensamientos, lo cual implicará adicionalmente una modificación del modo en que padres y docentes se vinculan con el menor, y del tipo de exigencias que les imponen.

\section{Conclusión}

Los datos de este estudio permiten concluir que, si bien en el segundo ciclo de la escolaridad primaria se observaron valores bajos y moderadamente bajos de perfeccionismo, existen casos en situación de riesgo psicológico por su exacerbación. De las manifestaciones perfeccionistas evaluadas, la que más sobresale es la autorientada, particularmente referida al ámbito escolar. Le siguen en orden de importancia el perfeccionismo prescrito y el perfeccionismo orientado a otros. El perfil más vulnerable corresponde a varones de 4to año, de escuelas públicas de la provincia de Misiones.

Estos resultados aportan insumos importantes para el próximo desarrollo de estrategias y recursos orientados a los niños, pero también y especialmente para trabajar con los docentes y padres a fin de concientizarlos sobre la problemática del perfeccionismo insano y su posible prevención y abordaje desde el ámbito escolar.

\section{Referencias}

Accordino, D. B., Accordino, M. P., \& Slaney, R. B. (2000). An investigation of perfectionism, mental health, achievement, and achievement motivation in adolescents. Psychology in 
the Schools, 37(6), 535-545. doi:10.1002/1520-6807(200011)37:6<535::AIDPITS6>3.0.CO;2-O

Adams, L. A., \& Govender, K. (2008). "Making a Perfect Man”: traditional masculine ideology and perfectionism among adolescent boys. South African Journal of Psychology, 38(3), 551-562. doi:10.1177/008124630803800309

Affrunti, N. W., \& Woodruff-Borden, J. (2014). Perfectionism in Pediatric Anxiety and Depressive Disorders. Clinical Child and Family Psychology Review, 17(3), 299-317. doi:10.1007/s10567-014-0164-4.

Barrow, J. C., \& Moore, C. A. (1983). Group interventions with perfectionistic thinking. The Personnel and Guidance Journal, 61(10), 612-615. doi:10.1111/j.21644918.1983.tb00008.x

Bas, A. U. (2011). Perfectionism Dimensions in Children: Associations with Anxiety, Life Satisfaction, and Academic Achievement. Egitim ve Bilim, 36(162), 261. Extraído desde https://search.proquest.com/openview/50bb9a17f8480e63643679a101be4297/1?pqorigsite $=$ gscholar $\& \mathrm{cbl}=1056401$

Blatt, S. J. (1995). The destructiveness of perfectionism: Implications for the treatment of depression. American psychologist, 50(12), 1003-1020. doi:10.1037/0003066X.50.12.1003

Bolsi, A., Paolasso, P., \& Longhi, F. (2006). El norte grande argentino entre el progreso y la pobreza. Población y sociedad, 12-13, 231-270. Extraído desde http://www.redalyc.org/html/3869/386939739007/

Bong, M., Hwang, A., Noh, A., \& Kim, S. (2014). Perfectionism and Motivation of Adolescents in Academic Contexts. Journal of Educational Psychology, 106(3), 711-729. doi:10.1037/a0035836

Chemisquy, S. (2018). Revisión teórica sobre el perfil cognitivo del perfeccionismo desadaptativo. Apuntes Universitarios, 8(1), 16-40. doi: 10.17162/au.v8i1.177

De Rosa, L. (2012). Factores mantenedores del perfeccionismo desadaptativo o clínico. En IV Congreso Internacional de Investigación y Práctica Profesional en Psicología, XIX Jornadas de Investigación, VIII Encuentro de Investigadores en Psicología del MERCOSUR. Facultad de Psicología - Universidad de Buenos Aires, Buenos Aires. 3942. Extraído desde https://www.aacademica.org/000-072/210.pdf

DiBartolo, P. M., Frost, R. O., Chang, P., LaSota, M., \& Grills, A. E. (2004). Shedding light on the relationship between personal standards and psychopathology: The case for contingent self-worth. Journal of Rational-Emotive and Cognitive-Behavior Therapy, 22(4), 237-250. doi:10.1023/B:JORE.0000047310.94044.ac

DiBartolo, P. M., \& Varner, S. P. (2012). How children's cognitive and affective responses to a novel task relate to the dimensions of perfectionism. Journal of Rational-Emotive and Cognitive-Behavior Therapy, 30(2), 1-15. doi:10.1007/s10942-011-0130-8

Dunn, J. G. H., Gotwals, J. K., \& Causgrove Dunn, J. (2005). An examination of the domain specificity of perfectionism among intercollegiate student-athletes. Personality and Individual Differences, 38(6), 1439-1448. doi:10.1016/j.paid.2004.09.009

Dunn, J. G., Gotwals, J. K., Dunn, J. C., \& Syrotuik, D. G. (2006). Examining the relationship between perfectionism and trait anger in competitive sport. International Journal of Sport and Exercise Psychology, 4(1), 7-24. doi:10.1080/1612197X.2006.9671781 
Egan, S. J., Wade, T. D., Shafran, R., \& Antony, M. M. (2014). Cognitive-behavioural treatment of perfectionism. Nueva York: Guilford Press.

Elizathe, L., Murawski, B., Custodio, J., \& Rutsztein, G. (2012). Riesgo de trastorno alimentario en niños escolarizados de Buenos Aires: su asociación con perfeccionismo. Revista Mexicana de Trastornos Alimentarios, 3, 106-120. Extraído desde http://www.scielo.org.mx/pdf/rmta/v3n2/v3n2a4.pdf

Ellis, A. (2002). The role of irrational beliefs in perfectionism. In G. L. Flett \& P. L. Hewitt (Eds.), Perfectionism: Theory, research, and treatment (pp. 217-229). Washington, DC, US: American Psychological Association.

Flett, G. L., \& Hewitt, P. L. (2013). Disguised distress in children and adolescents "Flying under the radar": Why psychological problems are underestimated and how schools must respond. Canadian Journal of School Psychology, 28(1), 12-27. doi:10.1177/0829573512468845

Flett, G. L., Hewitt, P. L., Boucher, D. J., Davidson, L. A., \& Munro, Y. (2000). The childadolescent perfectionism scale: Development, validation, and association with adjustment. Unpublished manuscript, University of British Columbia.

Flett, G. L., Hewitt, P. L., Oliver, J. M., \& Macdonald, S. (2002). Perfectionism in children and their parents: A developmental analysis. In G. L. Flett \& P. L. Hewitt (Eds.), Perfectionism: Theory, research, and treatment (89-132). Washington, DC, US: American Psychological Association. doi:10.1037/10458-004

García-Fernández, J. M., Inglés, C. J., Vicent, M., Gonzálvez, C., Gómez-Núñez, M. I., \& Poveda-Serra, P. (2016). Perfeccionismo durante la infancia y la adolescencia. Análisis bibliométrico y temático (2004-2014). Revista Iberoamericana de Psicología y Salud, 7(2), 79-88. doi:10.1016/j.rips.2016.02.001

George, D., \& Mallery, P. (2010). SPSS for Windows Step by Step: A Simple Guide and Reference 18.0 Update. Boston, MA: Allyn \& Bacon/Pearson.

Gilman, R., Adams, R., \& Nounopoulos, A. (2010). The Interpersonal Relationships and Social Perceptions of Adolescent Perfectionists. Journal of Research on Adolescence, 21(2), 505-511. doi:10.1111/j.1532-7795.2010.00689.x

Gonzálvez, C., Sanmartín, R., García-Fernández, J. M., Inglés, C. J., \& Vicent, M. (2016). Perfeccionismo socialmente prescrito como predictor del alto rechazo a la escuela. INFAD Revista de Psicología, 1(1), 25-32. Extraído desde http://hdl.handle.net/10045/53927

Gonzálvez Macia, C., Vicent Juan, M., Inglés, C. J., Lagos San Martín, N., García Fernández, J. M., \& Martínez, M. C. (2015). Diferencias en perfeccionismo socialmente prescrito en función del rechazo escolar. INFAD Revista de Psicología, 1(1), 455-462. doi:10.17060/ijodaep.2015.n1.v1.47

Hewitt, P. L., Caelian, C. F., Flett, G. L., Sherry, S. B., Collins, L., \& Flynn, C. A. (2002). Perfectionism in children: associations with depression, anxiety and anger. Personality and Individual Differences, 32(6), 1049-1061. doi:10.1016/S0191-8869(01)00109-X

Hewitt, P. L., \& Flett, G. L. (1991). Perfectionism in the self and the social contexts: Conceptualization, assessment, and association with psychopatology. Journal of personality and social psychology, 60(3), 456-470. doi:10.1037/0022-3514.60.3.456 
Hewitt, P. L., Flett, G. L., Sherry, S. B., \& Caelian, C. (2006). Trait perfectionism dimensions and suicide behavior. In T. E. Ellis (Ed.), Cognition and Suicide. Theory, Research and Therapy (pp. 215-235). Washington DC: American Psychological Association.

Instituto Nacional de Estadística y Censos (INDEC) (2018). Incidencia de la pobreza y la indigencia en 31 aglomerados urbanos. Primer semestre de 2018. Informes Técnicos, 2 (188), 1-15. Extraído desde https://www.indec.gob.ar/uploads/informesdeprensa/eph_pobreza_01_18.pdf.

Kotman, T., \& Ashby, J. (2000). Perfectionistic Children and Adolescents: Implications for School Counselors. Professional School Counseling, 3(3), 182-188.

Krüger, N., \& Formichella, M. M. (2012). Escuela pública y privada en Argentina: una comparación de las condiciones de escolarización en el nivel medio. PERSPECTIVAS. Revista de Análisis de Economía, Comercio y Negocios Internacionales, 6(1), 113-144. Extraído desde http://publicaciones.eco.uaslp.mx/VOL9/Paper04-6(1).pdf

Lozano Fernández, L. M., García Cueto, E., Martín Vázquez, M., \& Lozano González, L. (2012). Desarrollo y validación del Inventario de Perfeccionismo Infantil (IPI). Psicothema, 24(1), 149-155. Extraído desde http://www.psicothema.com/pdf/3992.pdf

Macedo, A., Soares, M. J., Amaral, A. P., Nogueira, V., Madeira, N., Roque, C., ... \& Pereira, A. T. (2015). Repetitive negative thinking mediates the association between perfectionism and psychological distress. Personality and Individual Differences, 72 , 220-224.

McArdle, S. (2009). Exploring domain-specific perfectionism. Journal of Personality, 78(2), 493-508. doi:10.1111/j.1467-6494.2010.00624.x

Ministerio de Salud, Organización Panamericana de la Salud, \& Organización Mundial de la Salud (2017). Indicadores Básicos Argentina 2017. Extraído desde http://www.deis.msal.gov.ar/wp-content/uploads/2018/04/IndicadoresBasicos2017.pdf

Morris, L., \& Lomax, C. (2014). Review: Assessment, development, and treatment of childhood perfectionism: a systematic review. Child and Adolescent Mental Health, 19(4), 225234. doi:10.1111/camh.12067

Nounopoulos, A., Ashby, J. S., \& Gilman, R. (2006). Coping resources, perfectionism, and academic performance among adolescents. Psychology in the Schools, 43(5), 613-622. doi:10.1002/pits.20167

Nugent, S. A. (2000). Perfectionism Its Manifestations and Classroom-Based Interventions. The Journal of Secondary Gifted Education, 11(4), 215-221. doi:10.4219/jsge-2000-630

Ocampo, M. (2008). Percepción del perfeccionismo parental como predictor del perfeccionismo infantil. Tesis de grado, Universidad Adventista del Plata, Entre Ríos, Argentina.

Oros, L. B. (2000). Incidencia de las prácticas parentales sobre las creencias y los modos de afrontamiento del niño. Tesis doctoral. Universidad Nacional de San Luis, Facultad de Ciencias Humanas, San Luis, Argentina.

Oros, L. B. (2003). Medición del perfeccionismo infantil: desarrollo y validación de una escala para niños de 8 a 13 años de edad. Revista Iberoamericana de Diagnóstico y Evaluación Psicológica, 16(2), 99-112. Extraído desde http://www.aidep.org/03_ridep/R16/R166.pdf 
Oros, L. B. (2005). Implicaciones del perfeccionismo infantil sobre el bienestar psicológico: Orientaciones para el diagnóstico y la práctica clínica. Anales de psicología. 21(2), 294303. Extraído desde http://www.redalyc.org/articulo.oa?id=16721211

Oros, L. B., Iuorno, O., \& Serppe, M. (2017). Child Perfectionism and its relationship with Personality, Excessive Parental Demands, Depressive Symptoms and Experience of Positive Emotions. Spanish Journal of Psychology, 20(9), 1-13. doi:10.1017/sjp.2017.9

Oros, L. B., Serppe, M., Chemisquy, S., \& Ventura-León, J. (2018). Construcción de una escala para evaluar el perfeccionismo infantil desadaptativo en su dimensión social. Manuscrito presentado para su publicación.

Oros, L. B., \& Vargas-Rubilar, J. (2016). Perfeccionismo infantil: Normalización de una escala argentina para su evaluación. Acción Psicológica, 13(2), 117-126. doi:10.5944/ap.13.2.17822

Pamies, L., \& Quiles, Y. (2014). Perfectionism and risk factors for the development of eating disorders in Spanish adolescents of both genders. Anales de Psicología/Annals of Psychology, 30(2), 620-626. doi:10.6018/analesps.30.2.158441

Richaud de Minzi, M. C. (2007). Fortalecimiento de recursos cognitivos, afectivos, sociales y lingüísticos en niñez en riesgo ambiental por pobreza: un programa de intervención. En M. C. Richaud de Minzi \& M. Ison (Eds.). Avances en Investigación en Ciencias del Comportamiento en Argentina, Vol. 1, Niñez en riesgo por pobreza (pp. 147-176). Mendoza: Editorial de la Universidad del Aconcagua.

Rimm, S. (2007). What's wrong with perfect? clinical perspectives on perfectionism and underachievement. Gifted Education International, 23(3), 246-253. doi:10.1177/026142940702300305

Roselló, J., \& Maysonet, M. (2015). Sintomatología depresiva, actitudes disfuncionales, perfeccionismo y auto-concepto en un grupo de adolescentes puertorriqueños. Revista Puertorriqueña de Psicología, 13(1), 225-241. Extraído desde https://dialnet.unirioja.es/servlet/articulo?codigo $=4895930$

Roxborough, H. M., Hewitt, P. L., Kaldas, J., Flett, G. L., Caelian, C. M., Sherry, S., \& Sherry, D. L. (2012). Perfectionistic self-presentation, socially prescribed perfectionism, and suicide in youth: A test of the perfectionism social disconnection model. Suicide and Life-Threatening Behavior, 42(2), 217-233. doi:10.1111/j.1943-278X.2012.00084.x

Samaniego, C. (2004). Prevalencia de trastornos psíquicos en población escolar de 6 a 11 años de edad. En XI Jornadas de Investigación. Facultad de Psicología-Universidad de Buenos Aires. Extraído desde https://www.aacademica.org/000-029/190.pdf

Samaniego, C. (2008). El Child Behavior Checklist: su estandarización en población urbana argentina. Revista de Psicología 4(8). Extraído desde http://bibliotecadigital.uca.edu.ar/repositorio/revistas/child-behavior-cheklistestandarizacion-samaniego.pdf

Schruder, C. R., Curwen, T., \& Sharpe, G. W. B. (2014). Perfectionistic students: Contributing factors, impacts, and teacher strategies. British Journal of Education, Society \& Behavioural Science, 4(2), 139-155. doi:10.9734/BJESBS/2014/5532

Serppe, M., Chemisquy, S., \& Oros, L. B. (2016). Evaluación del perfeccionismo insano en varones y niñas de 9 a 12 años: ¿quiénes están en mayor riesgo de desarrollarlo? Trabajo presentado en el X Congreso Argentino de Salud Mental y III Congreso Regional de la World Federation for Mental Health, Buenos Aires. 
Shafran, R., Cooper, Z., \& Fairburn, C. G. (2002). Clinical perfectionism: A cognitive behavioural analysis. Behaviour Research and Therapy, 40(7), 773-791. doi:10.1016/S0005-7967(01)00059-6.

Sherry, S. B., Mackinnon, S. P., \& Gautreau, C. M. (2016). Perfectionists don't play nicely with others: Expanding the social disconnection model. In F. M. Sirois \& D. S. Molnar (Eds.), Perfectionism, health, and well-being (pp. 225-243). New York: Springer.

Shrive, F. M., Stuart, H., Quan, H., \& Ghali, W. A. (2006). Dealing with missing data in a multi-question depression scale: a comparison of imputation methods. BMC medical research methodology, 6(1), 57. doi:10.1186/1471-2288-6-57

Siegle, D., \& Schuler, P. A. (2000). Perfectionism differences in gifted middle school students. Roeper Review, 23(1), 39-44. doi:10.1080/02783190009554060

Sondergeld, T. A., Schultz, R. A., \& Glover, L. K. (2007). The need for research replication: An example from studies on perfectionism and gifted early adolescents. Roeper Review, 29(5), 19-25. doi:10.1080/02783193.2007.11869220

Stoeber, J., \& Rambow, A. (2007). Perfectionism in adolescent school students. Relations with motivation, achievement, and well-being. Personality and Individual Differences, 42(7), 1379-1389. doi:10.1016/j.paid.2006.10.015

Stoeber, J., \& Stoeber, F. S. (2009). Domains of perfectionism: Prevalence and relationships with perfectionism, gender, age, and satisfaction with life. Personality and Individual Differences, 46, 530-535. doi:10.1016/j.paid.2008.12.006

Stoeber, J., Noland, A. B., Mawenu, T. W., Henderson, T. M., \& Kent, D. N. (2017). Perfectionism, social disconnection, and interpersonal hostility: Not all perfectionists don't play nicely with others. Personality and Individual Differences, 119, 112-117. doi:10.1016/j.paid.2017.07.008

Vargas Rubilar, J. (Agosto, 2015). Estilos parentales y contexto social: la relación con los hijos desde la perspectiva de los padres. En el Simposio: Avances en investigación en recursos socio-cognitivos y afectivos en la niñez media y tardía. Presentado en la $V$ Reunión Nacional y IV Encuentro Internacional de la AACC, $2^{\circ}$ Congreso Argentino de Biología del Comportamiento COMPORTA 2015, Tucumán, Argentina.

Vargas Rubilar, J., Lemos, V., \& Richaud, M. C. (2017). Programa de fortalecimiento parental en contextos de vulnerabilidad social: una propuesta desde el ámbito escolar. Interdisciplinaria, $\quad 34(1), \quad$ 157-172. Extraído desde http://www.scielo.org.ar/pdf/interd/v34n1/v34n1a10.pdf

Vicent, M., Aparicio-Flores, M. P., Inglés, C. J., Gómez-Núñez, M., Fernández-Sogorb, A., \& Aparisi-Sierra, D. (2017a). Perfeccionismo infantil: diferencias en función del sexo y la edad. International Journal of Developmental and Educational Psychology, 3(1), 15 24. doi:10.17060/ijodaep.2017.n1.v3.970

Vicent, M., Inglés, C. J., Sanmartín, R., Gonzálvez, C., \& García-Fernández, J. M. (2017). Perfectionism and aggression: Identifying risk profiles in children. Personality and Individual Differences, 112, 106-112. doi:10.1016/j.paid.2017.02.061

Vuyk, M. A. (2015). Perfeccionismo, autopresentación perfeccionista, y síntomas depresivos en adolescentes paraguayos según género y grado. Interamerican Journal of Psychology, 49(2), 153-163. Extraído desde http://www.redalyc.org/articulo.oa?id=28446019003 
